\title{
Extreme warm absorber variability in the Seyfert galaxy Mrk 704
}

\author{
G. Matt ${ }^{1}$, S. Bianchi ${ }^{1,2}$, M. Guainazzi ${ }^{3}$, A. L. Longinotti ${ }^{4}$, M. Dadina ${ }^{5}$, V. Karas $^{6}$, G. Malaguti ${ }^{5}$, G. Miniutti ${ }^{7}$, \\ P. O. Petrucci ${ }^{8}$, E. Piconcelli ${ }^{9}$, and G. Ponti ${ }^{10}$ \\ ${ }^{1}$ Dipartimento di Fisica, Università degli Studi Roma Tre, via della Vasca Navale 84, 00146 Roma, Italy \\ e-mail: matt@fis.uniroma3.it \\ 2 INAF - Osservatorio Astronomico di Brera, via E. Bianchi 46, 23907 Merate, Italy \\ 3 European Space Astronomy Center of ESA, Apartado 50727, 28080 Madrid, Spain \\ 4 M.I.T. Kavli Institute for Astrophysics and Space Research, Cambridge, USA \\ 5 INAF-IASF Bologna, via Gobetti 101, 40129 Bologna, Italy \\ 6 Astronomical Institute, Academy of Sciences, Bocni II 1401, 14131 Prague, Czech Republic \\ 7 Centro de Astrobiología (CSIC-INTA) LAEFF, PO Box 78, Villanueva de la Cañada, Madrid 28691, Spain \\ ${ }^{8}$ UJF-Grenoble 1/CNRS-INSU, Institut de Planétologie et d'Astrophysique de Grenoble (IPAG) UMR 5274, 38041 Grenoble, \\ France \\ 9 INAF - Osservatorio Astronomico di Roma, via Frascati 33, 00040 Monteporzio Catone, Roma, Italy \\ 10 School of Physics and Astronomy, University of Southampton, Highfield, Southampton SO17 1BJ, UK
}

Received 5 January 2011 / Accepted 5 July 2011

\begin{abstract}
Context. In about half of the Seyfert galaxies, the X-ray emission is absorbed by an optically thin, ionized medium, the so-called "warm absorber", whose origin and location is still a matter of debate.

Aims. The aim of this paper is to constrain the warm absorber further by studying its variability.

Methods. We analyzed the X-ray spectra of a Seyfert 1 galaxy, Mrk 704, which was observed twice, three years apart, by XMM-Newton

Results. The spectra were well fitted with a two-zones absorber, which possibly covers the source only partially. The parameters of the absorbing matter - column density, ionization state, covering factor - changed significantly between the two observations. Possible explanations for the more ionized absorber are a torus wind (the source is a polar scattering one) or, in the partial covering scenario, an accretion disk wind. The less ionized absorber is possibly composed of orbiting clouds in the surroundings of the nucleus, similarly to what was already found in other sources, most notably NGC 1365.
\end{abstract}

Key words. galaxies: active - X-rays: galaxies - X-rays: individuals: Mrk 704

\section{Introduction}

Absorption from ionized matter in the X-ray spectrum of AGN (the so-called warm absorber) was discovered many years ago (Halpern 1984). Since then, our understanding of this matter has advanced through many studies. We know now that it is present in about half of Seyfert galaxies (e.g. Reynolds 1997) and that it is photoionized. Warm absorbers are also known to vary, and indeed the first discovered absorber was variable (Halpern 1984). The location of the warm absorber (or, indeed, of the absorbers, because more than one ionizing zone is often found) is, however, largely uncertain. There is some evidence for its origin as a wind from the dusty torus envisaged in unification models for Seyfert galaxies (Blustin et al. 2005), but cases in which an origin from the disk seems to be preferred do also exist (Krongold et al. 2007).

Mrk 704 is a local $(z=0.029234)$ Seyfert 1.2 galaxy (Veron-Cetty \& Veron 2010), bright enough in X-rays to be detected by Swift/BAT (Ajello et al. 2008). In this paper we report on extreme warm absorber variability on yearly time scales, revealed by two XMM-Newton observations, and possible variability on monthly time scales from short Swift/XRT observations.

The paper is organized as follows: in Sect. 2 we report on the XMM-Newton observations and data reduction, while the relative data analysis is discussed in Sect. 3. Section 4 presents the analysis of the Swift and ASCA observations, while the results are summarized and discussed in Sect. 5.

\section{XMM-Newton: observations and data reduction}

XMM-Newton observed Mrk 704 twice, on 2005-10-21 (oBsID: 0300240101) and on 2008-11-02 (oBSID: 0502091601). In both cases, EPIC pn and MOS were in Small Window mode (apart from MOS2 in the first observation, which was in Full Frame mode), which ensures that no significant pile-up is present, as verified with the EPATPLOT tool, in the pn detectors (the only one used for the analysis). Mrk 704 is by far the brightest source in the field of view.

Data were reduced with SAS 10.0.0, using calibration files generated on 2010-6-11. Screening for intervals of flaring particle background was consistently made by extracting radii in an iterative process based on the procedure to maximize the signalto-noise ratio described by Piconcelli et al. (2005). After this process, the net exposure time was of about 15 and $68 \mathrm{ks}$ for the 2005 and the 2008 observation, respectively, adopting extraction radii of 36 and 40 arcsec and patterns 0 to 4 . The background spectra were extracted from source-free circular regions with a radius of 50 arcsec. The same regions were used for the timing analysis. Spectra were binned to oversample the instrumental resolution by at least a factor of 3 and to have no less 

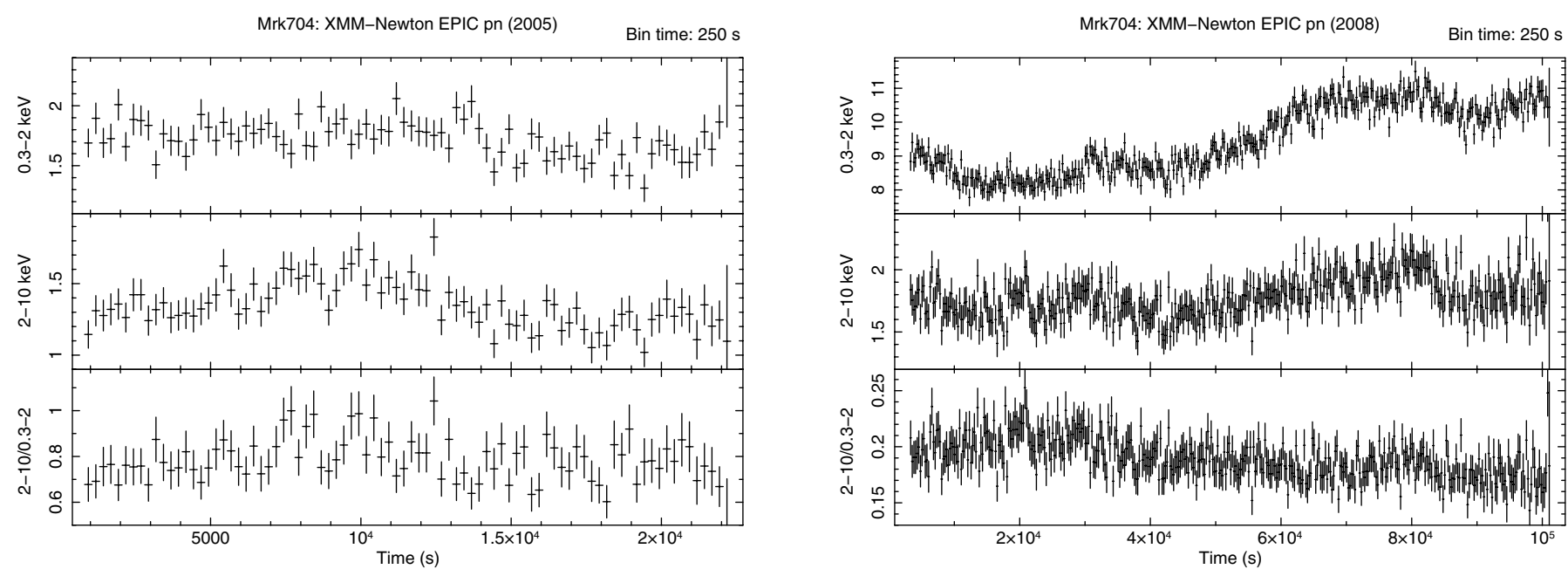

Fig. 1. $0.3-2 \mathrm{keV}$ (upper panel), 2-10 keV (middle panel) and the hard-to-soft ratio (lower panel) light curves for the first (left panels) and second (right panels) XMM-Newton observations.
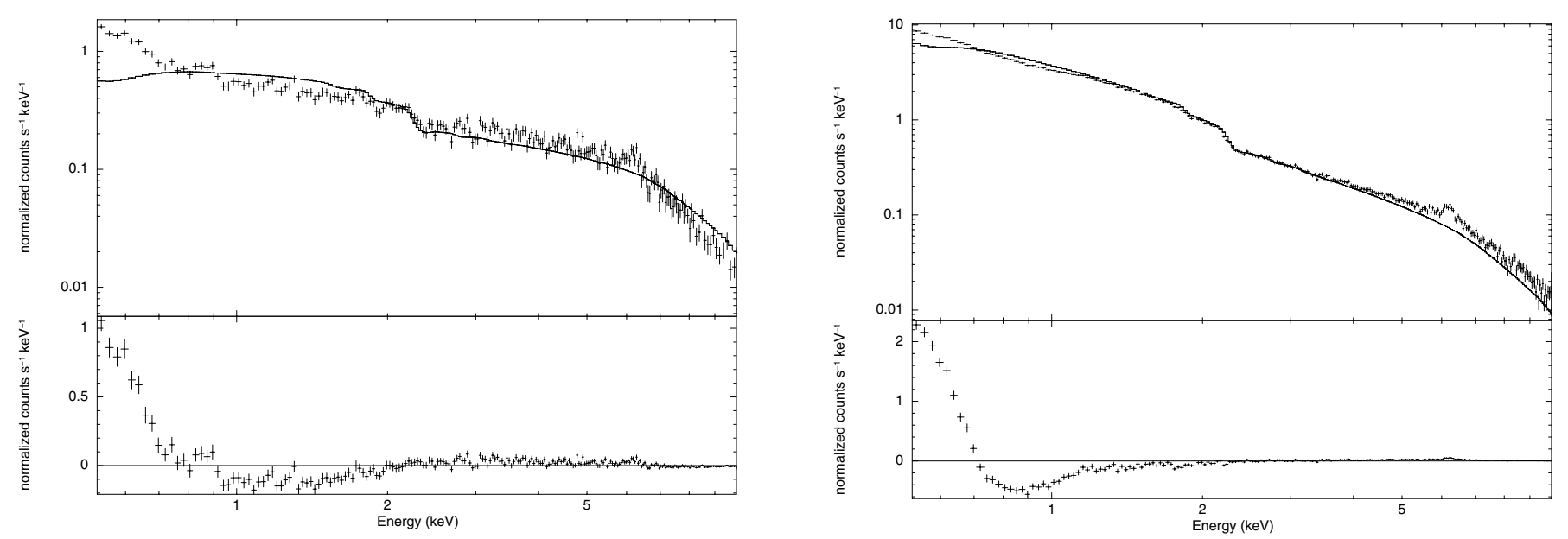

Fig. 2. Spectrum and residuals for the first (left) and second (Right) XMM-Newton observations when fitted with a simple power law absorbed by Galactic interstellar matter.

than 30 counts in each background-subtracted spectral channel. The latter requirement allows us to use the $\chi^{2}$ statistics as a goodness-of-fit-test. For the reflection grating spectrometers (RGS) source and background spectra were extracted with standard procedures, adopting the data reduction pipeline RGSPROC, and choosing the optical nucleus of Mrk 704 as the reference point for the attitude solution.

\section{XMM-Newton: data analysis}

We will use only data from the EPIC pn and the RGS camera in this paper, because according to the latest release of the EPIC pn redistribution and the RGS contamination model (May 2010) their cross-calibration is as good as 3\% (A. Pollock, priv. comm.).

In Fig. 1 the $2-10 \mathrm{keV}$ (upper panel), $0.3-2 \mathrm{keV}$, (middle panel) and the $(2-10 \mathrm{keV}) /(0.3-2 \mathrm{keV})$ hardness ratio (HR) light curves are shown for the first (left) and the second (right) observations. Intra-observation variability in the count rates is apparent in both cases. While in the first observation no clear variation in the HR is present, in the second observation an anticorrelation of the hardness ratio with the flux is evident, indicating that the source becomes softer when brighter. Therefore, while for the first observation we will use the time-integrated spectrum only, for the second observation we will also make a time-resolved spectral analysis.

Spectral analysis was performed using the XSPEC package, version 12.6.0. Unless specifically stated, all errors refer to the $90 \%$ confidence level for one interesting parameter.

We begin with the time-averaged analysis of both observations. In Fig. 2 the best-fit models and residuals are shown, assuming a simple power law absorbed by the Galactic column density in the direction of the source $\left(2.97 \times 10^{20} \mathrm{~cm}^{20}\right.$, Kalberla et al. 2005). Not surprisingly the fits are clearly bad, given the extremely simple model. From the residuals one can make out a soft excess, a broad absorption trough between about 1 and $2 \mathrm{keV}$ - indicative of obscuration by ionised gas - and an iron line at $6.4 \mathrm{keV}$.

Comparing the spectra of the two observations (Fig. 3), a huge difference in the soft part of the spectrum is clear, suggesting a much stronger absorption in the first observation. Above a few $\mathrm{keV}$, instead, the two spectra are pretty similar (see also Fig. 4). 
G. Matt et al.: Extreme warm absorber variability in the Seyfert galaxy Mrk 704

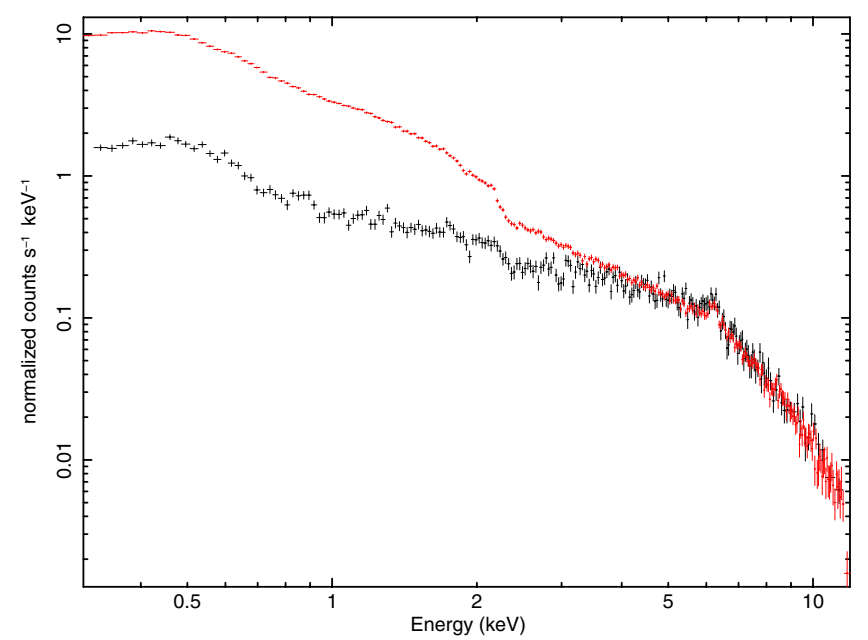

Fig. 3. Two XMM-Newton spectra shown together for comparison. The soft X-ray flux in the first observation is much lower than in the second observation, while above $5 \mathrm{keV}$ the two spectra are almost indistinguishable.

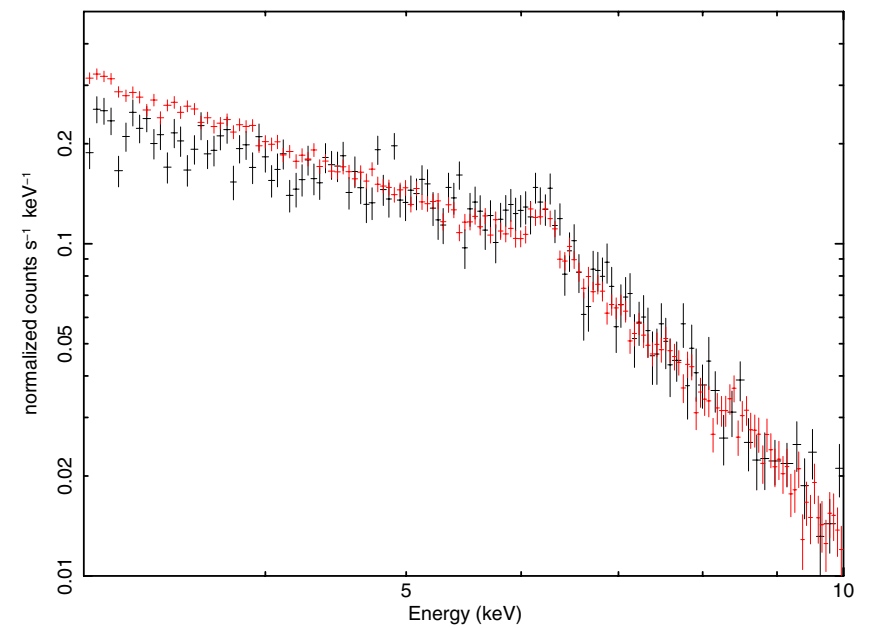

Fig. 4. Hard X-ray spectra of the two XMM-Newton observations shown together for comparison.

\subsection{The iron line}

We first concentrated on the iron line. To this end we limited the bandwidth to 5-10 keV, where the soft excess and the warm absorber do not have an important impact on the spectral modeling. More specifically, the choice of $5 \mathrm{keV}$ as the lower limit of the band was motivated on one hand by the necessity to have the entire line within the band (which is verified a posteriori), and on the other hand to avoid any effect of the warm absorber. In fact, a fit with a simple power law gives very different values - especially for the first observation - of the photon index depending on the lower limit of the band, which stabilizes only above $5 \mathrm{keV}$. The photon index steepens when the lower limit increases, a clear indication of absorption at low energies.

We fitted the 5-10 keV spectrum with a power law, a Compton reflection component (PEXRAV model in XSPEC, parameter $R$ - the solid angle subtended by the reflecting matter to the nuclear source in units of $2 \pi$ - fixed to 1 ), and an iron line, at first modeled with a narrow Gaussian at $6.4 \mathrm{keV}$ ( $\sigma$ fixed to 0 ). Results are summarized in Table 1, where best-fit spectral parameters for this and the subsequent models are reported. In both observations, the statistical quality of the fit is poor, and
Table 1. Iron line results.

\begin{tabular}{lcc}
\hline \hline Parameters & Obs. 1 & Obs. 2 \\
\hline Narrow Gaussian & & \\
$F_{\text {line }}\left(10^{-5} \mathrm{cgs}\right)$ & $1.17_{-0.37}^{+0.36}$ & $0.95_{-0.15}^{+0.17}$ \\
$E W(\mathrm{eV})$ & 85 & 72 \\
$\chi^{2} /$ d.o.f. & $77.2 / 73$ & $113.3 / 93$ \\
\hline Broad Gaussian & & \\
$\sigma(\mathrm{eV})$ & $143_{-59}^{+66}$ & $116_{-35}^{+42}$ \\
$F_{\text {line }}\left(10^{-5} \mathrm{cgs}\right)$ & $2.18_{-0.69}^{+0.73}$ & $1.51_{-0.30}^{+0.32}$ \\
$E W(\mathrm{eV})$ & 162 & 119 \\
$\chi^{2} /$ d.o.f. & $66.2 / 72$ & $91.4 / 92$ \\
\hline Diskline & & \\
$r_{\text {in }}\left(r_{\mathrm{g}}\right)$ & $52_{-24}^{+80}$ & $>80$ \\
$\theta$ & $20_{-6}^{+5}$ & $22_{-7}^{+20}$ \\
$F_{\text {line }}\left(10^{-5} \mathrm{cgs}\right)$ & $2.24_{-0.61}^{+1.01}$ & $1.32_{-0.25}^{+0.21}$ \\
$E W(\mathrm{eV})$ & 175 & 113 \\
$\chi^{2} /$ d.o.f. & $62.6 / 71$ & $90.7 / 91$ \\
\hline Diskline + Narrow Gaussian & \multicolumn{3}{c}{} \\
$F_{\text {line,disk }}\left(10^{-5}\right.$ cgs $)$ & $2.66_{-1.28}^{+2.00}$ & $1.49_{-0.58}^{+0.57}$ \\
$E W_{\text {disk }}(\mathrm{eV})$ & 208 & 127 \\
$F_{\text {line,NG }}\left(10^{-5}\right.$ cgs $)$ & $0.68_{-0.42}^{+0.46}$ & $0.64_{-0.20}^{+0.19}$ \\
$E W_{\text {NG }}(\mathrm{eV})$ & 44 & 52 \\
$\chi^{2} /$ d.o.f. & $66.7 / 72$ & $96.0 / 92$ \\
\hline & &
\end{tabular}

residuals are evident around the line, suggesting it is resolved. Indeed, leaving $\sigma$ free to vary, the quality of the fit significantly improves (see Table 1), with $\sigma$ of $100-150 \mathrm{eV}$, corresponding to a FWHM velocity 2-3 times higher than that of the broad line regions (about $5000 \mathrm{~km} \mathrm{~s}^{-1}$, Stirpe 1990). Letting the centroid energy of the line free to vary instead does not improve the quality of the fit.

The most likely broadening mechanism is therefore matter rotation in an accretion disk. We consequently substituded the Gaussian with the DISKLINE model (valid for Schwarschild metric) with the outer radius fixed to 1000 gravitational radii and the emissivity index to -2.5 . The rest-frame line energy was again fixed to $6.4 \mathrm{keV}$. The fits are of comparable statistical quality to those obtained with a broad Gaussian. The moderate width of the line translates into a quite extended inner radius (much more extended than the innermost stable orbit) and into a small inclination angle.

On the other hand, it is possible that the line is actually a blend of a narrow line (originating e.g. in the torus) and of a relativistic line. We therefore fitted the spectrum with a combination of the two, fixing the inner radius of the relativistic line to six gravitational radii for simplicity (the innermost stable orbit for Schwarschild metric) and the inclination angle to 30 degrees. The fit is again good, and we cannot really distinguish between the different models on statistical grounds. (A fit of similar quality is obtained if a LAOR model is used for the line, again with the inner radius fixed to the innermost stable orbit.) We limit ourselves to note that a relativistic component seems to be required.

Comparing the two observations, there is marginal evidence for a stronger (and broader) broad component of the line in the first observation (see Fig. 4). The quality of the data is, however, not good enough to explore this possible line variability in more detail.

For both observations, and for all line models, the power law photon index is around 1.7-1.8. 

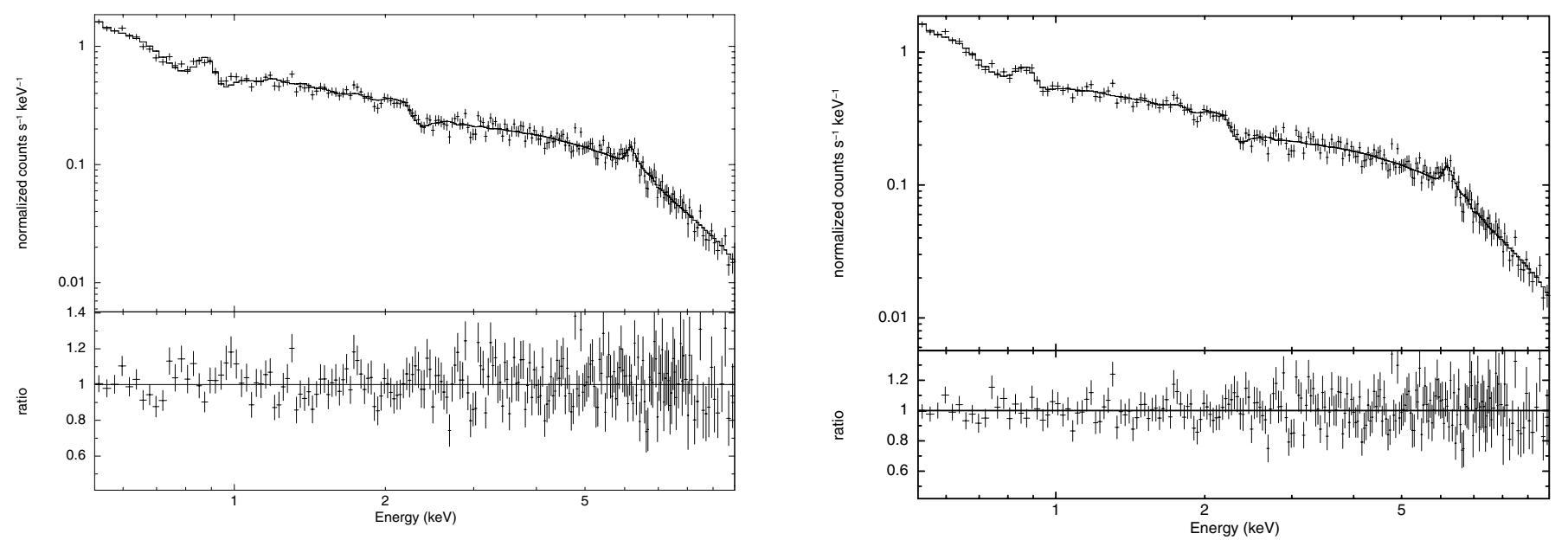

Fig. 5. Obs 1: best-fit model and data/model ratio for the two zones model. Left panel: full covering absorbers. Right panel: partial covering absorbers.

\subsection{The warm absorber}

We now study the broad band $(0.5-10 \mathrm{keV})$ spectra of the source. With the residuals to a simple power law model in mind (Fig. 2), we added to the model already used to study the iron line (adopting for simplicity a broad Gaussian for the line), a second power law to model the soft excess, and one or more warm absorber region. The warm absorber was modeled with the ZXIPCF model, based on the XSTAR photoionization code. The main limitations of the model are a fixed ionizing continuum (a power law with photon index of 2) and fixed element abundances (equal to solar). The ionization parameter is $\xi=L / n R^{2}$. The results are summarized in Table 2. For the first observation, the addition of an emission line around $0.9 \mathrm{keV}$ (likely to be identified with the neon IX He-like triplet) was also necessary, as also suggested by the RGS analysis. In fact, a line at $0.903 \pm$ $0.001 \mathrm{keV}$ (corresponding to the forbidden line of the triplet) with a flux of $1.69( \pm 0.72) \times 10^{-5} \mathrm{ph} \mathrm{cm}^{-2} \mathrm{~s}^{-1}$ was found in the RGS spectrum of obs. 1 (see below).

\subsubsection{The first observation}

Let us first discuss the first observation, where the absorption is much stronger. A single ionization zone is insufficient, but the addition of a second zone improves the statistical quality of the fit significantly, even if not dramatically. Moreover, in the single zone model $\Gamma_{\mathrm{s}}$ (the photon index of the soft power law) is unrealistically steep, while $\Gamma_{\mathrm{h}}$ (the photon index of the hard power law) is much flatter than the value found in the hard band.

The addition of the second zone improves the quality of the fit which however remains not fully satisfactory, however. Visual inspection of the data/model ratio (see Fig. 5), however, shows that this is due mostly to noise rather than to unfitted features, even if some wiggles are present around the Neon line. Indeed, an improvement $\left(\Delta \chi^{2}=-16\right)$ of the fit is obtained by letting the width of the line free to vary when a value of $48 \mathrm{eV}$ is found. This may suggest a blend with other lines (mostly iron L), too faint to be individually detected by the RGS. However, it is also possible that the broadening of the line is an artifact to correct for a too strong iron UTA in the model (this may occur e.g. if the iron abundance is lower than solar). Unfortunately, with the present data we cannot proceed much further in this analysis.

No improvement is found by adding a third absorption region.
Table 2. Warm absorber results.

\begin{tabular}{|c|c|c|}
\hline Parameters & Obs. 1 & Obs. 2 \\
\hline \multicolumn{3}{|l|}{1 Zone } \\
\hline$\Gamma_{\mathrm{s}}$ & $10.0_{-0.1}^{+0.0(*)}$ & $4.55_{-0.25}^{+0.42}$ \\
\hline$\Gamma_{\mathrm{h}}$ & $1.45_{-0.04}^{+0.05}$ & $1.87_{-0.02}^{+0.02}$ \\
\hline$N_{\mathrm{H}}\left(10^{22} \mathrm{~cm}^{-2}\right)$ & $5.16_{-0.34}^{+0.49}$ & $0.15_{-0.03}^{+0.02}$ \\
\hline$\xi(\mathrm{cgs})$ & $122_{-8}^{+9}$ & $39_{-5}^{+8}$ \\
\hline$E_{\text {line }}(\mathrm{keV})$ & $0.900_{-0.008}^{+0.005}$ & - \\
\hline$F_{\text {line }}\left(10^{-5} \mathrm{cgs}\right)$ & $4.12_{-0.48}^{+0.45}$ & - \\
\hline$\chi^{2} /$ d.o.f. & $295.1 / 208$ & $421.9 / 230$ \\
\hline \multicolumn{3}{|l|}{2 Zones } \\
\hline$\Gamma_{\mathrm{s}}$ & $5.4_{-2.1}^{+2.4}$ & $3.53_{-0.14}^{+0.21}$ \\
\hline$\Gamma_{\mathrm{h}}$ & $1.64_{-0.07}^{+0.06}$ & $1.79_{-0.02}^{+0.02}$ \\
\hline$N_{\mathrm{H}, 1}\left(10^{22} \mathrm{~cm}^{-2}\right)$ & $0.14_{-0.09}^{+0.08}$ & $0.085_{-0.008}^{+0.007}$ \\
\hline$\xi_{1}(\mathrm{cgs})$ & $0.39_{-0.18}^{+0.58}$ & $5.1_{-1.1}^{+0.09}$ \\
\hline$N_{\mathrm{H}, 2}\left(10^{22} \mathrm{~cm}^{-2}\right)$ & $6.85_{-0.63}^{+0.57}$ & $1.63_{-0.56}^{+1.15}$ \\
\hline$\xi_{2}(\mathrm{cgs})$ & $139_{-13}^{+13}$ & $1750_{-190}^{+205}$ \\
\hline$E_{\text {line }}(\mathrm{keV})$ & $0.904_{-0.007}^{+0.005}$ & - \\
\hline$F_{\text {line }}\left(10^{-5} \mathrm{cgs}\right)$ & $4.57_{-0.53}^{+0.49}$ & - \\
\hline$\chi^{2} /$ d.o.f. & $256.4 / 206$ & $279.4 / 228$ \\
\hline \multicolumn{3}{|c|}{2 Zones, partial covering } \\
\hline$\Gamma_{\mathrm{s}}$ & $6.7_{-3.2}^{+3.3(*)}$ & $2.71_{-0.08}^{+0.14}$ \\
\hline$\Gamma_{\mathrm{h}}$ & $1.71_{-0.05}^{+0.06}$ & $1.18_{-0.45}^{+0.33}$ \\
\hline$N_{\mathrm{H}, 1}\left(10^{22} \mathrm{~cm}^{-2}\right)$ & $0.10_{-0.05}^{+1.00}$ & $0.75_{-0.06}^{+0.08}$ \\
\hline$\xi_{1}(\mathrm{cgs})$ & $0.29_{-0.19}^{+1.90}$ & $2.24_{-0.29}^{+0.38}$ \\
\hline Covering Factor (1) & $>0.45$ & $0.56_{-0.03}^{+0.02}$ \\
\hline$N_{\mathrm{H}, 2}\left(10^{22} \mathrm{~cm}^{-2}\right)$ & $8.8_{-1.0}^{+1.8}$ & $13.3_{-2.5}^{+1.9}$ \\
\hline$\xi_{2}(\mathrm{cgs})$ & $97_{-11}^{+15}$ & $122_{-17}^{+64}$ \\
\hline Covering Factor (2) & $0.84_{-0.04}^{+0.04}$ & $0.38_{-0.13}^{+0.11}$ \\
\hline$E_{\text {line }}(\mathrm{keV})$ & $0.895_{-0.014}^{+0.011}$ & - \\
\hline$F_{\text {line }}\left(10^{-5} \mathrm{cgs}\right)$ & $2.50_{-0.75}^{+0.78}$ & - \\
\hline$\chi^{2} /$ d.o.f. & $234.3 / 204$ & $230.2 / 226$ \\
\hline
\end{tabular}

Notes. ${ }^{(*)}$ The asterisk indicates that a parameter is pegged to one of its limits.

We tried to fit the warm absorber also with "home-made" tables built using the cLOUDY photoionization code. The results were similar and are therefore not reported here. 

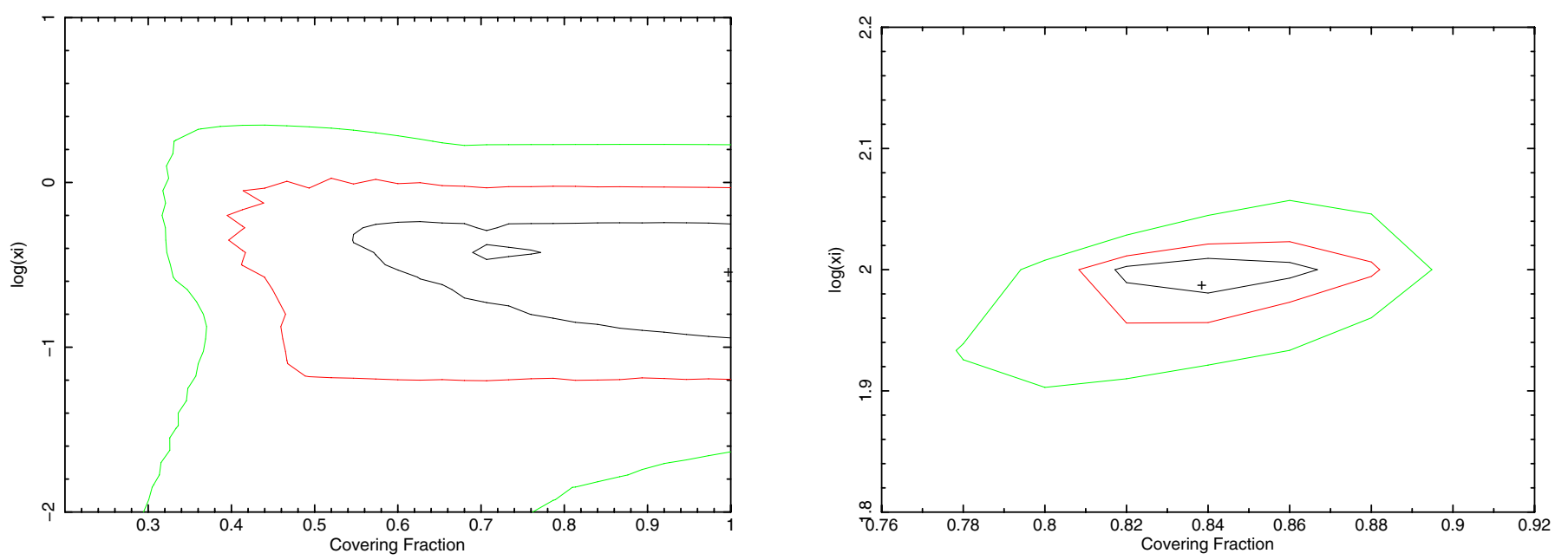

Fig. 6. Obs 1: contour plots (ionization parameters vs. covering factors) for the low (left panel) and high (right panel) ionization absorbers. The three curves represent the $67 \%, 90 \%$ and $99 \%$ confidence levels.

Table 3. Velocities of the warm absorber regions.

\begin{tabular}{lcc}
\hline \hline Models & Obs. 1 & Obs. 2 \\
\hline 2 Zones & & \\
$v_{\text {low }}$ & $-3900_{-6500}^{+3300 \mathrm{~km} \mathrm{~s}^{-1}}$ & $-190 \pm 210 \mathrm{~km} \mathrm{~s}^{-1}$ \\
$v_{\text {high }}$ & $+2650_{-870}^{+9300} \mathrm{~km} \mathrm{~s}^{-1}$ & $-100 \pm 250 \mathrm{~km} \mathrm{~s}^{-1}$ \\
\hline 2 Zones, partial covering & & \\
$v_{\text {low }}$ & - & $-580 \pm 340$ \\
$v_{\text {high }}$ & $+3000_{-1800}^{+1500} \mathrm{~km} \mathrm{~s}^{-1}$ & $+140 \pm 300 \mathrm{~km} \mathrm{~s}^{-1}$ \\
\hline
\end{tabular}

As a consistency check, we then fitted the RGS data with the best-fit model (all parameters fixed) described above. Spectra were rebinned to obtain at least 10 counts per $\operatorname{bin}^{1}$. The fit is reasonable $\left(\chi^{2} /\right.$ d.o.f. $\left.=333.4 / 278\right)$, with several, but not individually significant features apparent in the residuals apart from the Ne IX line already included in the spectrum. An improvement $\left(\chi^{2} /\right.$ d.o.f. $\left.=296.2 / 269\right)$ is found letting the model parameters free to vary. The best-fit parameters, however, are consistent within the errors with those found in the EPIC-pn analysis, and the abovementioned residuals are still there.

We then let the velocities of the absorbing zones free to vary to account for possible inflows/outflows. A better fit is found $\left(\chi^{2} /\right.$ d.o.f. $\left.=281.2 / 267\right)$ with an inflow velocity of

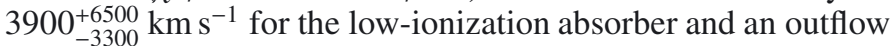
velocity of $2650_{-870}^{+9300} \mathrm{~km} \mathrm{~s}^{-1}$ for the high-ionization absorber (see Table 3 where "low" and "high" refers to the low- and highionization components, respectively, and negative velocities indicate inflows).

Back to the EPIC-pn analysis, a slight improvement $\left(\Delta \chi^{2}=-8\right)$ is also found substituting the soft power law with a disk thermal component (DISKBB model in XSPEC). The temperature is around $160 \mathrm{eV}$, but the normalization value is unphysical, corresponding to about $4 \times 10^{5} \mathrm{~km}$ for the inner radius (the Schwarzschild radius for the 100 million solar-masses black hole estimated for Mrk 704, Wang et al. 2009, is about one thousand times larger).

\footnotetext{
1 As a check that the rebinning was not altering the fit results, we fitted the RGS spectra also with no rebinning and the Cash quality-of-the-fit statistics, and obtained no significantly different results.
}

A slightly worse fit $\left(\Delta \chi^{2}=14\right)$ is instead found if an ionized reflection model (REFLIONX, Ross \& Fabian 2005) is adopted (with or without relativistic blurring) for the soft X-ray component. The power law index is pegged to the lower possible value, 1.4 .

A moderate improvement $\left(\Delta \chi^{2}=-22\right)$ is found when the absorbers are partial. Best-fit covering factors of 1 (but loosely constrained) and 0.85 are found for the colder and warmer ionization zones, respectively (see Table 2). All other parameters are similar to those found in the full-absorbers fit. The best-fit model and data/model ratio can be found in Fig. 5. In Fig. 6 the contour plots (ionization parameter vs. covering factor) are shown. For the low-ionization absorber, a full covering is always acceptable, while at the $99 \%$ confidence level a very low-ionization (almost neutral) partial absorber is possible. The two parameters are much better determined for the high-ionization absorber, with no significant correlation between them.

Regarding the RGS, a check similar to that described above for the full covering scenario was also performed in the partial covering case. With all parameters fixed, the fit is worse than for the full absorbers $\left(\chi^{2} /\right.$ d.o.f. $\left.=356.5 / 278\right)$. A slightly better fit is instead found letting the parameters free to vary $\left(\chi^{2} /\right.$ d.o.f. $\left.=283.7 / 267\right)$, again with the best-fit parameters consistent within the errors with those found in the EPIC-pn analysis. The feature-like residuals are still visible (see Fig. 7). Letting the velocities of the absorbers free to vary, we found a marginal improvement of the quality of the fit $\left(\chi^{2} /\right.$ d.o.f. $\left.=279.2 / 265\right)$, with an outflow velocity of $3000_{-1800}^{+1500} \mathrm{~km} \mathrm{~s}^{-1}$ for the high-ionization absorber, while the velocity of the low-ionization absorber is basically undetermined (Table 3).

The value of $\Gamma_{\mathrm{h}}$ is lower than that found in the iron line analysis. This, together with the relatively high $\chi^{2}$, may indicate that the warm absorber model we are adopting is not fully adequate (e.g., the absorption regions may be more structured that single ionization zones, or metal abundances be different than solar).

For simplicity, the iron line is modeled with a broad Gaussian in the broad band fitting. No significant changes in the line parameters are found with respect to the hard X-ray band fits described above.

The 0.5-2 (2-10) keV observed flux is 1.53(10.1) $\times$

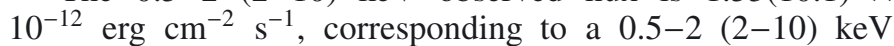
luminosity of $1.17(2.34) \times 10^{43} \mathrm{erg} \mathrm{s}^{-1}$, after correcting for absorption. 


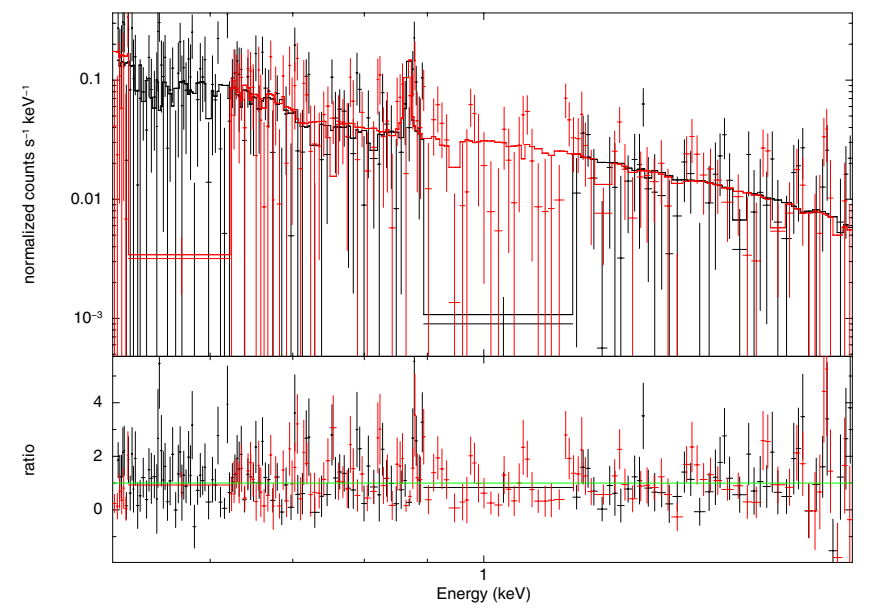

Fig. 7. Reflection grating spectrometer best-fit spectra and data/model ratio for the first observation. Fit with partial covering absorbers.

\subsubsection{The second observation}

Owing to the much weaker absorption and the absence of the neon emission line and despite the better statistics, the analysis of the second observation was easier. In this case the addition of a second zone is definitely required as well.

Inspection of the data/model ratio (see Fig. 8) shows, however, that some features are still remaining, even if the one at about $1.8-2 \mathrm{keV}$ is very likely of instrumental origin. Particularly prominent is the feature around $1 \mathrm{keV}$, again suggesting a possible problem in the fitting of the iron transitions. The addition of a third absorbing zone does not solve this problem.

For this observation we also found similar results with the cLoudy-based, home-made tables. A significantly worse fit is found instead by substituting the soft power law either with a disk thermal component or with an ionized reflection model.

We then fitted the RGS data with the best-fit model (all parameters fixed) described above. Given the much better statistics with respect to the first observation, spectra were rebinned to have at least 50 counts per bin $^{2}$. The fit is reasonable $\left(\chi^{2} /\right.$ d.o.f. $\left.=856.8 / 679\right)$. An improvement $\left(\chi^{2} /\right.$ d.o.f. $\left.=814.4 / 672\right)$ is found letting the model parameters free to vary. The best fit parameters, however, are consistent within the errors with those found in the EPIC-pn analysis. Inspection of the residuals indicates the possible presence of features, but the only significant one (at the $99 \%$ confidence level) is an emission line with a centroid energy of $1.114 \pm 0.002 \mathrm{keV}$ - to be possibly identified with Fe XVII L lines - with a flux of $1.80( \pm 0.75) \times$ $10^{-5} \mathrm{ph} / \mathrm{cm}^{2} / \mathrm{s}^{-1}$. However, no such line is found in the EPIC-pn data (upper limit of $3.6 \times 10^{-6} \mathrm{ph} / \mathrm{cm}^{2} / \mathrm{s}^{-1}$ ). Finally, no significant inflow/outflow velocity is found, with upper limits of a few hundreds $\mathrm{km} \mathrm{s}^{-1}$ (Table 3 ).

A significant improvement $\left(\Delta \chi^{2}=-49\right)$ is found with partial absorbers. Best-fit covering factors of 0.56 (but loosely constrained) and 0.38 are found for the colder and warmer ionization zones, respectively (see Table 2). Both absorbers are now much thicker and slightly less ionized. The power law indices are much harder; in particular, the $\Gamma_{\mathrm{h}}$ is now 1.18 (even if loosely constrained), a value unusually low for Seyfert galaxies. However, it must be noted that by fixing $\Gamma_{\mathrm{h}}=1.8$ a good fit is still obtained $\left(\chi^{2}=243.0 / 227\right)$, with the parameters of the

\footnotetext{
2 Also in this case, fits to the unbinned spectra provide substantially the same results.
}

less ionized absorber almost unchanged, while the more ionized absorber becomes even more ionized and thicker $\left(N_{\mathrm{H}, 2} \sim 4 \times\right.$ $10^{23} \mathrm{~cm}^{-2}, \xi_{2} \sim 230$, covering factor of 0.27$)$. The best-fit model and data/model ratio can be found in Fig. 8. In Fig. 9 we show the contour plots (ionization parameter vs covering factor). For the low-ionization absorber the two parameters are almost uncorrelated, while for the high-ionization absorber they are anticorrelated, indicating a certain degree of degeneracy in the model.

The usual check with the RGS was performed next. The fits are better than those obtained with the full absorbers, and reflect the same improvement as was found in the EPIC-pn spectrum. With all parameters fixed, a $\chi^{2} /$ d.o.f. $=821.6 / 679$ is found, while with the parameters free to vary we found $\chi^{2} /$ d.o.f. $=752.0 / 670$. The best-fit parameters are consistent within the errors with those found in the EPIC-pn analysis. The spectrum and data/model ratio can be seen in Fig. 10. Finally, no significant inflow/outflow velocity is found for the highionization absorber (upper limits of a few hundreds $\mathrm{km} \mathrm{s}^{-1}$ ), while for the low-ionization absorber a marginally significant inflow velocity of $580 \pm 340 \mathrm{~km} \mathrm{~s}^{-1}$ is obtained (Table 3 ).

For the second observation, the $0.5-2(2-10) \mathrm{keV}$ observed flux is $7.62(11.2) \times 10^{-12} \mathrm{erg} \mathrm{cm}^{-2} \mathrm{~s}^{-1}$, corresponding to a $0.5-2$ $(2-10) \mathrm{keV}$ luminosity of $2.34(2.26) \times 10^{43} \mathrm{erg} \mathrm{s}^{-1}$, after correcting for absorption.

During the revision of the present work, we became aware of a paper by Laha et al. (2011) dealing with the analysis of the second XMM-Newton observation of Mrk 704. The results of their analysis are qualitatively similar to ours, the quantitative differences probably mostly related to the somewhat different spectral model adopted ${ }^{3}$. The most important qualitative difference is that Laha et al. found the two warm absorbers both outflowing, while in our analysis no significant inflow/outflow is found, with only marginal evidence for an inflow of the low-ionization absorber in the partial covering scenario.

\subsubsection{Comparison between the two observations}

A comparison between the results of the fits to the two spectra of the two observations shows, as is obvious from Fig. 3, stronger overall absorption in the first observation. Assuming full covering absorbing zones, the column density decreases for both observations (by a factor of about 2 for the colder absorber, and of about 4 for the warmer absorber). The ionization parameter increases from the first to the second observation. If the absorbers are partial, the colder absorber is thicker, warmer and with a lower covering factor in the second observation, while for the warmer zone the most significant difference is the covering factor, which is again much lower in the second observation (the column density and the ionization parameter both slightly increase).

The detection of a neon emission line in the first observation only cannot be entirely ascribed to the stronger absorption (which would make a constant line more visible by contrast). Indeed, simulations show that a line with this flux should have been detected also in the second observation (even if the upper limit to such a line, about $10^{-5}$, is marginally consistent with

\footnotetext{
3 The main difference in the modeling is that they included a partial, neutral intrinsic absorber, while keeping full coverage for the ionized absorbers. If we include this in the two full absorbers model, we find a slightly better fit, but significantly worse than the one with two partial ionized absorbers however. If we include this in the first observation, a fit very similar to that with two partial ionized absorbers is found, which is not surprising because the lower ionization zone is almost neutral in that observation (see Fig. 6).
} 
G. Matt et al.: Extreme warm absorber variability in the Seyfert galaxy Mrk 704
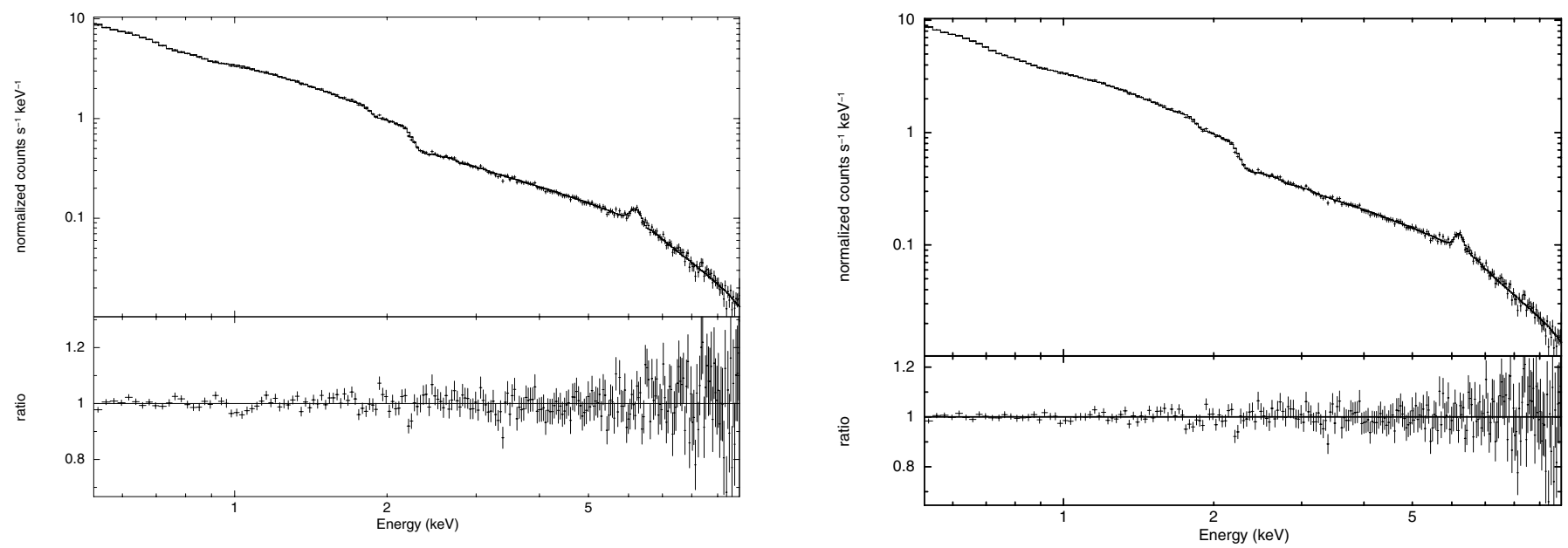

Fig. 8. Obs 2: best-fit model and data/model ratio for the two zones model. Left panel: full covering absorbers. Right panel: partial covering absorbers.
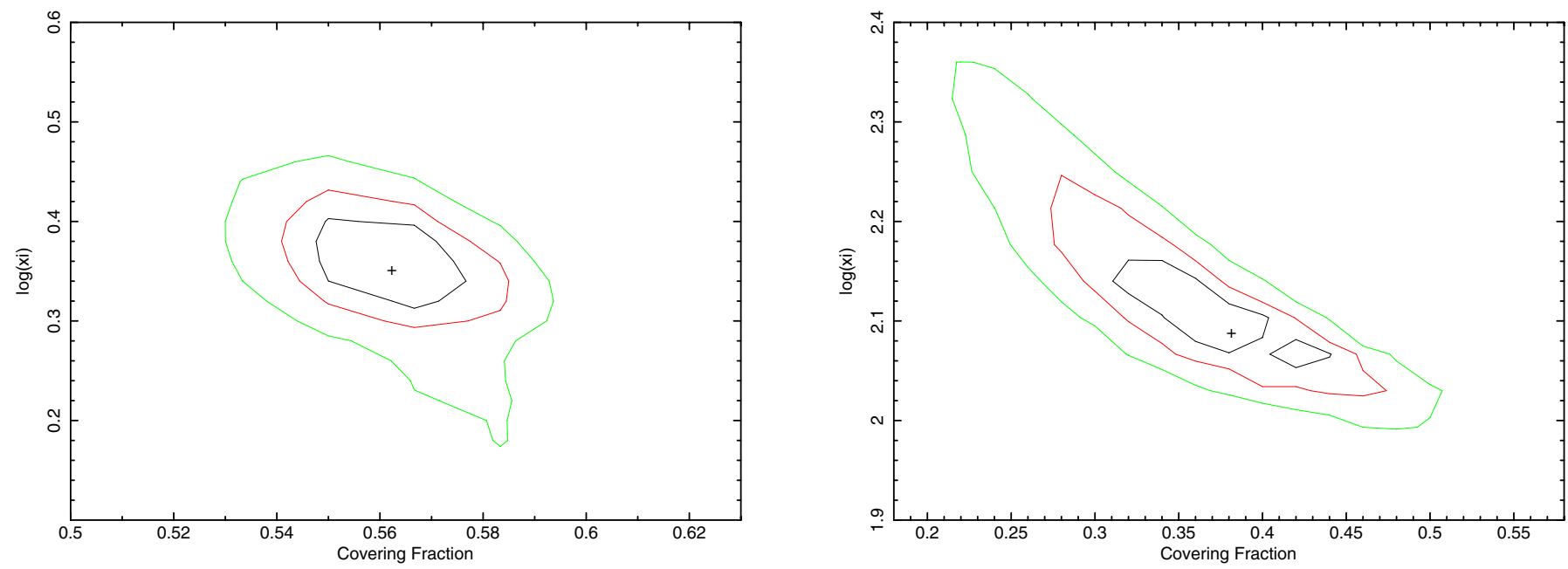

Fig. 9. Obs 1: contour plots (ionization parameters vs. covering factors) for the low- (left panel) and high- (right panel) ionization absorbers. The three curves represent the $67 \%, 90 \%$ and $99 \%$ confidence levels.

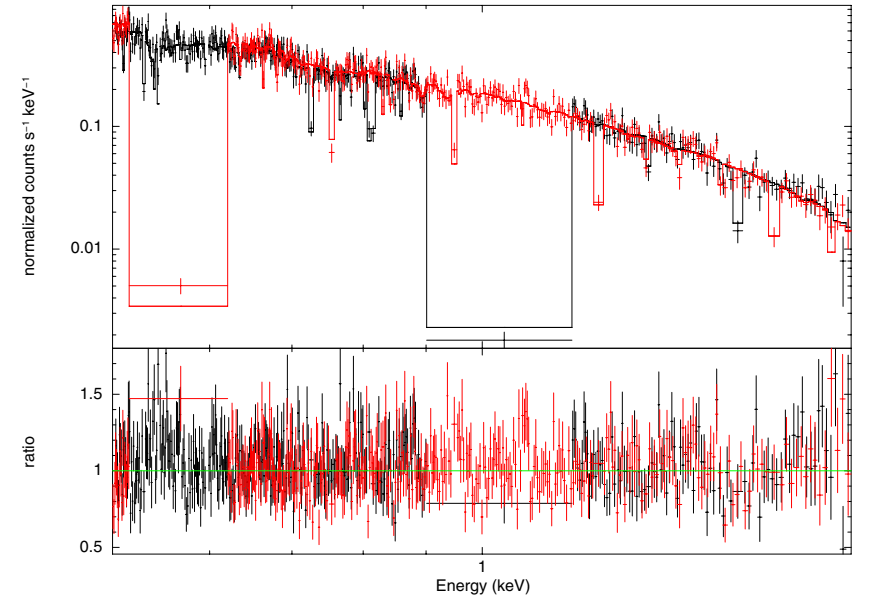

Fig. 10. Reflection Gratings Spectrometer best-fit spectra and data/model ratio for the second observation. Fit with partial covering absorbers.

the value found in the RGS in the first observation). Therefore, an intrinsic variability of the line intensity is required, which is possibly related to a different covering factor of the absorbing/reflecting clouds.

The question is then: what drove this considerable variation of the warm absorber over three years? The hard 2-10 X-ray luminosity stayed approximately the same, while the soft 0.5-2 keV luminosity was higher by a factor 2 in the second observation (even if the reconstructed values, given the high absorption, are fairly uncertain), which may - in part at least explain the higher ionization parameters. However, the different values in the two observations of the column densities and of the neon line suggest that also variations in the general properties (size, distance, covering fraction, etc.) of the circumnuclear medium have occurred as well.

\subsubsection{Time-dependent analysis of the second observation}

Because of its intra-observation spectral variability (see Fig. 1), we divided the second observation into two parts, corresponding to the first $40 \mathrm{ks}$ (obsA here-in-after) and to the remaining part of the observation (obsB), respectively. The three spectra (obsA, obsB and total) are shown in Fig. 11. A reasonable fit $\left(\chi^{2} /\right.$ d.o.f. of $266.2 / 228$ and $274.5 / 235$ for obsA and obsB, respectively) is obtained by fixing all spectral parameters to 
Table 4. Swift/XRT results.

\begin{tabular}{lcccc}
\hline \hline Parameters & Obs. 1 & Obs. 2 & Obs. 3 & Obs 5 \\
\hline Date & $2006-01-06$ & $2006-06-14$ & $2006-09-28$ & $2007-01-21$ \\
XRT exp. time (s) & 671 & 2258 & 5602 & 1750 \\
$\Gamma$ & 2 (fixed) & $2.00_{-0.16}^{+0.21}$ & $1.88_{-0.12}^{+0.11}$ & 2 (fixed) \\
$N_{\mathrm{H}}\left(10^{22} \mathrm{~cm}^{-2}\right)$ & $12_{-8}^{+8}$ & $0.85_{-0.57}^{+2.20}$ & $1.58_{-1.06}^{+1.35}$ & $<1.1$ \\
$\xi(\mathrm{cgs})$ & $95_{-81}^{+27}$ & $73_{-53}^{+325}$ & $123_{-97}^{+172}$ & $<450$ \\
Flux $(2-10 \mathrm{keV})\left(10^{-11} \mathrm{cgs}\right)$ & 0.64 & 1.14 & 0.88 & 0.87 \\
$\chi^{2} /$ d.o.f. & $0.31 / 5$ & $38.4 / 36$ & $49.1 / 54$ & $19.3 / 22$ \\
\hline
\end{tabular}

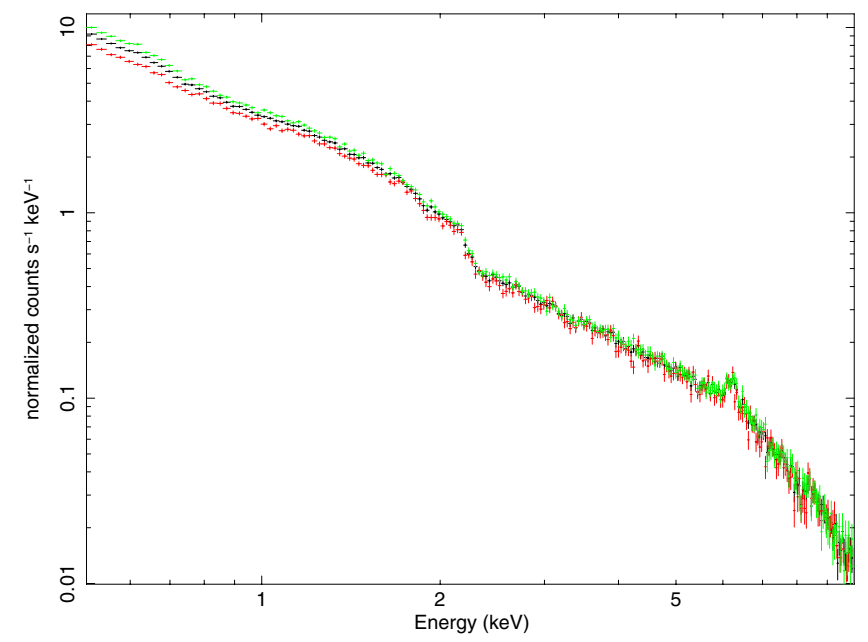

Fig. 11. Spectra from the first and second part of the second $X M M$-Newton observation shown together for comparison.

those found in the total spectrum (two-absorbing-zones model), leaving as free parameters only the normalizations of the two power laws. The observed spectral variability is then explained mainly in terms of a variation of the normalization of the soft power law. Other choices, like leaving instead the column density or the ionization parameter or the covering factor of the warm absorbers as a free parameter do not provide acceptable fits.

Leaving all main parameters free to vary, better fits are of course obtained $\left(\chi^{2} /\right.$ d.o.f. $=253.5 / 221$ and $265.2 / 228$ for obsA and obsB, respectively) but the parameter that suffered the highest variation is again the normalization of the soft power law, confirming the previous analysis.

\section{The Swift and ASCA observations}

The source was observed by Swift five times. On four occasions, it was also in the XRT field of view (see Table 4), while in the fourth observation it was outside. The four XRT spectra are shown in Fig. 12 along with the two XMM ones.

From the figure, spectral and flux variability is apparent between Swift observations (even if far less dramatic than that between the two XMM-Newton observations that were made with a gap of three years), implying a variability time scale of a few months or less. In Table 4 the results of the spectral fits are summarized. Because of the modest quality of the spectra, a good fit is already obtained with a single power law absorbed by a single zone, fully covering absorber (no improvement is found letting the covering of the absorber be partial). For the lesser quality first and last observations, we fixed the power law index to 2 ,

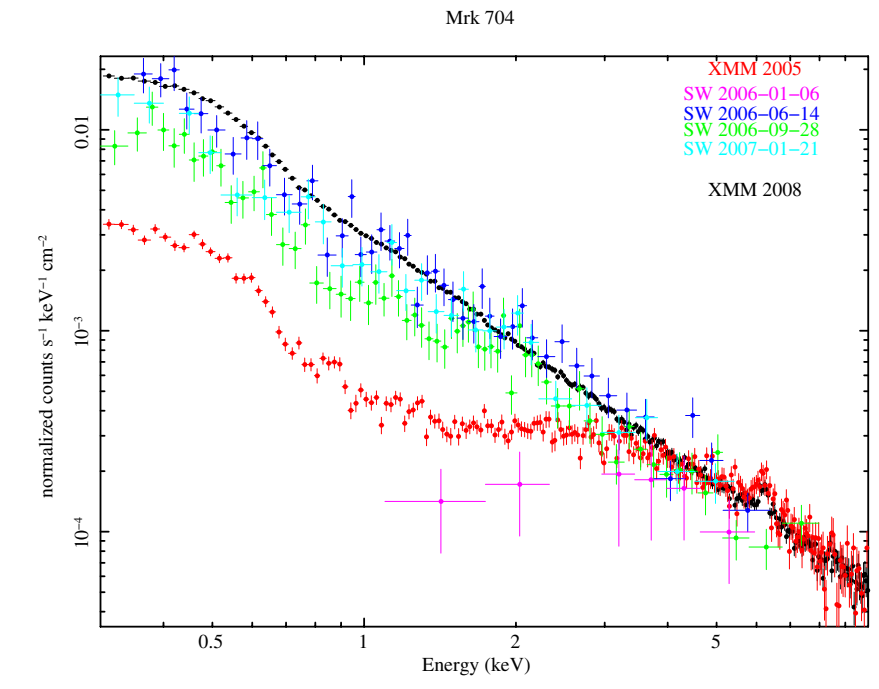

Fig. 12. Spectra of all XMM-Newton and Swift observations shown together for comparison.

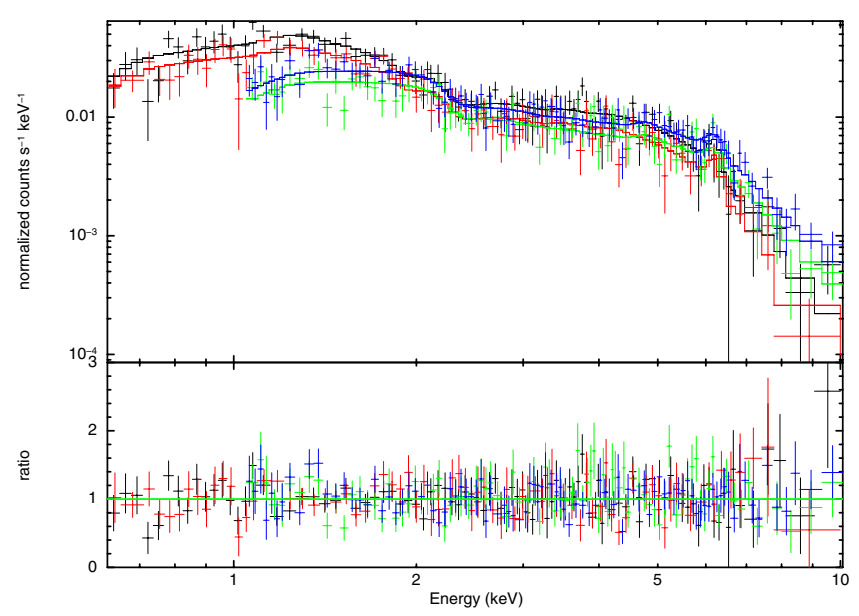

Fig. 13. Best-fit model and data/model ratio for the ASCA spectrum. See text for detail.

a value similar to those found in the second and third observations. The quality of the spectra is insufficient to establish if the variability is caused by a variation of the properties of the warm absorber, apart from the first observation, when a much higher absorption is visible.

Mrk 704 was also observed by ASCA on 1998-05-12, for about 35 ks exposure time. A good fit $\left(\chi^{2} /\right.$ d.o.f. $\left.=348.9 / 355\right)$ is already obtained with a single power law absorbed by a single zone, fully covering absorber (see Fig. 13). The source was in a lower flux state $\left(2-10 \mathrm{keV}\right.$ flux of about $5 \times 10^{-12} \mathrm{erg} \mathrm{cm}^{-2} \mathrm{~s}^{-1}$. 
The absorber has a column density of $5.6\left({ }_{-1.4}^{+3.5}\right) \times 10^{23} \mathrm{~cm}^{-2}$, higher than in any other observation, and an ionization parameter of $1430\left({ }_{-130}^{+270}\right)(\mathrm{cgs})$.

\section{Discussion and conclusions}

The Seyfert 1.2 galaxy Mrk 704 was observed twice by XMM-Newton with a gap of three years (first observation in October 2005, the second in November 2008). While the hard (>5 keV) spectrum was almost constant between the two observations (with a possible broadening/strenghtening of the iron line in the first observation), the soft part dramatically changed, showing much heavier absorption in the first observation. A strong neon IX line is also clearly present in the first observation, while it is not detected in the second one. The very similar hard X-ray spectrum in the two observations (the behavior of the intrinsic soft X-ray spectrum is more uncertain because of the strong absorption) together with the change in the column density suggest that the variations are caused by a change in the properties of the absorbing clouds. Dramatic changes of the column density and/or the ionization state of the absorber have already been observed in other sources, the most spectacular being NGC 4151 (e.g. Schurch \& Warwick 2002; de Rosa et al. 2007), NGC 7582 (Bianchi et al. 2009), and NGC 1365 (Risaliti et al. 2005, 2009). In the last two sources in particular considerable variations of the column density of the (cold) absorber occur on time scales as short as less than a day. Here we also observe a strong variation of the ionization state of the absorbers. Because the primary continuum (at least the hard one) stayed almost constant, this indicates a variation of the location and/or of the density of the clouds.

It is interesting to note that Mrk 704 is a "polar-scattering" Seyfert 1, i.e. a source with optical polarization aligned perpendicularly to the radio source axis, as is usually found in Seyfert 2s (Smith et al. 2004). Smith et al. suggest that in these sources the nucleus is seen through the edge of the torus.

We fitted the spectra with two absorbing regions, either fully or partially covering the primary emission. The improvement in the quality of the fit with the partial absorbers is only moderate for the first observation, but more significant in the second one. Because the results are somewhat different in the two cases, we discuss them separately.

With the full covering absorbers, both absorbing zones are found to be more ionized and to have a lower column density in the second observation (even if the difference in the column density for the low-ionization zone is only marginal). The RGS analysis of the first observation suggests a possible (but only marginally significant) inflow for the low-ionization, lowcolumn density absorber, and an outflow velocity (very poorly determined, with a $90 \%$ confidence level range between 1800 and $12000 \mathrm{~km} \mathrm{~s}^{-1}$ ) for the high-ionization, high-column density absorber. An ionized and unstable torus wind, as suggested by Smith et al., may indeed provide an explanation for the latter zone. No significant inflow/outflow is instead found for both absorbers in the second observation.

The partial covering scenario, even if providing better fits, is geometrically more demanding, requiring a size of the obscuring clouds on the same order as the emitting region. This naturally points to a much closer location of the obscuring clouds, as e.g. due to a radiatively-driven accretion disk wind (e.g. Proga 2003). Interestingly, the variability behavior of Mrk 704 closely resembles that of mini-BAL QSOs (Giustini et al. 2010), strengthening the disk wind scenario, at least for the more ionized absorber (the colder absorber may be composed of orbiting clouds as those found in NGC 1365, Risaliti et al. 2009). Interestingly, a possible outflow has been detected in the first observation (but not in the second, despite very similar column densities and ionization parameters).

The alternative hypothesis that the partial covering is mimicking the presence of a scattering component originating outside the absorbing region, is ruled out by the short-term variability of the X-ray emission.

Mrk 704 was also observed four times by Swift/XRT between January 2006 and January 2007. There is some spectral and flux variability between the observations, but the quality of the spectra is not sufficient to establish the nature of the variations.

The iron $\mathrm{K} \alpha$ line, studied with XMM, is broad, much broader than the optical broad lines but narrower than expected if the entire line would originate in the innermost accretion disk. However, if a narrow component (which seems to be almost ubiquitous in Seyfert galaxies) is added, then the remaining broad component is consistent with emission down to the innermost stable orbit, even if the quality of the data in not sufficient to constrain the spin of the black hole.

Acknowledgements. We thank the anonymous referee for her/his suggestions which helped to improve the clarity of the paper. We thank all the members of the FERO collaboration for useful discussions. G.M., S.B. and E.P. acknowledge financial support from ASI under grants I/088/06/0 and I/090/10/0/. P.O.P. acknowledges financial support from CNES and French GDR PCHE. G.P. acknowledges support from an EU Marie Curie Intra-European Fellowship under contract No. FP7-PEOPLE-2009-IEF-254279.

\section{References}

Ajello, M., Rau, A., Greiner, J., et al. 2008, ApJ, 673, 96

Bianchi, S., Piconcelli, E., Chiaberge, M., et al. 2009, ApJ, 695, 781

Blustin, A. J., Page, M. J., Fuerst, S. V., et al. 2005, A\&A, 431, 111

De Rosa, A., Piro, L., Perola, G. C., et al. 2007, A\&A, 463, 903

Giustini, M., Cappi, M., Chartas, G., et al. 2010, in Accretion and Ejection in

AGN: a Global View, ed. L. Maraschi, G. Ghisellini, R. Della Ceca, \& F. Tavecchio, ASP Conf. Ser., 427, 108

Halpern, J. P. 1984, ApJ, 281, 90

Kalberla, P. M. W., Burton, W. B., Hartmann, D., et al. 2005, A\&A, 440, 775

Krongold, Y., Nicastro, F., Elvis, M., et al. 2007, ApJ, 659, 1022

Laha, S., Dewangan, G. C., \& Kembhavi, A. K. 2011, ApJ, 734, 75

Piconcelli, E., Jimenez-Bailón, E., Guainazzi, M., et al. 2005, A\&A, 432, 15

Proga, D. 2003, ApJ, 585, 406

Risaliti, G., Elvis, M., Fabbiano, G., Baldi, A., \& Zezas, A. 2005, ApJ, 623, L93

Risaliti, G., Miniutti, G., Elvis, M., et al. 2009, ApJ, 696, 160

Reynolds, C. S. 1997, MNRAS, 286, 513

Ross, R. R., \& Fabian, A. C. 2005, MNRAS, 358, 211

Schurch, N. J., \& Warwick, R. S. 2002, MNRAS, 334, 811

Smith, J. E., Robinson, A., Alexander, D. M., et al. 2004, MNRAS, 350, 140

Stirpe, G. 1990, A\&AS, 85, 1049

Veron-Cetty, M.-P., \& Veron, P. 2010, A\&A, 518, 10

Wang, J., Mao, Y. F., \& Wei, J. Y. 2009, AJ, 137, 3388 